PUPT-1939

hep-th/0006132

\title{
String theory as a universal language
}

\author{
A.M. Polyakov \\ Joseph Henry Laboratories \\ Princeton University \\ Princeton, New Jersey 08544
}

\begin{abstract}
This article, based on the Klein lecture, contains some new results and new speculations on various topics. They include discussion of open strings in the AdS space, unusual features of D-branes, conformal gauge theories in higher dimensions. We also comment on the infrared screening of the cosmological constant and on the "brane worlds"
\end{abstract}

June 2000 


\section{Introduction}

String theory is a beautiful and dangerous subject. On one hand it is a top achievement of theoretical physics exploiting the most advanced and daring methods. On the other — without a guidance from the experiment it can easily degenerate into a collection of baroque curiosities, some kind of modern alchemy looking for philosopher's stone.

This danger can be somewhat reduced if we try to study string theory in connection with some concrete physical problem and then extrapolate the gained experience to the Planck domain unreachable by experiments. This is a well established strategy in theoretical physics. For example one can learn about the Cherenkov radiation while studying supersonic aerodynamics. And usually there is the "back-reaction": the technical progress at the frontier turns out to be helpful in solving the old problems. Thus, it is conceivable that string theory will provide us with the language for the future theoretical physics.

In this lecture I will examine a number of problems in which the language of string theory is appropriate and effective. We begin with the problem of quark confinement. The task here is to find the string description of the colorelectric flux lines emerging in QCD. Recently there has been a considerable progress in this field. Various aspects of it have been reviewed in [1,2]. I shall not review again these developments and instead concentrate on new results. After that we will discuss some general features of D-branes, conformal gauge theories in higher dimensions, and speculations concerning the cosmological constant.

\section{The image of gluons and zigzag symmetry}

As was explained in the above references, a string theory, needed to describe gauge fields in 4 dimensions, must be formulated in the $5 \mathrm{~d}$ space with the metric

$$
d s^{2}=d \varphi^{2}+a^{2}(\varphi) d \vec{x}^{2}
$$

This curved 5d space is a natural habitat for the color-electric flux lines. If apart from the pure gauge fields there are some matter fields in the theory, they must correspond to extra degrees of freedom on the world sheet. In 
some cases these extra degrees of freedom can be balanced so that the field theory $\beta$-function is equal to zero. These cases are the easiest ones, since the conformal symmetry of the field theory requires conformal symmetry of the string background and determines it completely:

$$
a(\varphi) \sim e^{\alpha \varphi}
$$

where $\alpha$ is some constant. After an obvious change of variables the metric takes the form

$$
d s^{2}=\sqrt{\lambda} y^{-2}\left(d y^{2}+d \vec{x}^{2}\right)
$$

where $\lambda$ is related to the coupling constant of field theory. When $\lambda \gg 1$ the curvature of this $5 \mathrm{~d}$ space is small and the $2 \mathrm{~d}$ sigma model describing the string in the above background is weakly coupled. This greatly simplifies the analyses and we will concentrate on this case. Our aim in this section will be to demonstrate that open strings in this background have some very unusual properties, allowing to identify them with gluons.

Before starting let us recall that at present we have two possible approaches to the question of field-string correspondence. None of them is fully justified but both have certain heuristic power. In the first approach one begins with a stack of D-branes describing a gauge theory and then replace the stack by its gravitational background. In the second approach one doesn't introduce D-branes and starts directly with the sigma model action, adjusting the background so that the boundary states of this string describe the gauge theory. The key principle here is the zigzag symmetry. This is a requirement that these boundary states consist of vector gluons (and matter fields, if present) and nothing else.

The situation is very unusual. Normally we have an infinite tower of states in both open and closed string sectors. Here we need a string theory in which the closed string sector contains an infinite number of states, while the open sector has a finite number of the field-theoretic states. Our first task will be to explore how this is possible.

To set the stage, let us remember how open strings are treated in the standard case [3]. One begins with the action

$$
S=\frac{1}{2} \int_{D}(\partial x)^{2}+i \int_{\partial D} A_{\mu} d x_{\mu},
$$


where $D$ is a unit disc, $\partial D$ - its boundary, and $A_{\mu}$ is a vector condensate of the open string states. The possible fields $A_{\mu}$ are determined from the condition that the functional integral

$$
Z[A]=\int D x e^{-S}
$$

is conformally invariant. The explicit form of this condition is derived by splitting

$$
x=c+z,
$$

where $c$ is a slow variable, while $z$ is fast, and integrating out $z$. Conformal invariance requires vanishing of the divergent counterterms and that restricts the background fields. It is convenient to integrate first the fields inside the disk with the fields at the boundary being fixed. That gives the standard boundary action

$$
S_{B}=\frac{1}{2} \int \frac{d u d v}{(u-v)^{2}}(x(u)-x(v))^{2}+i \int A_{\mu} d x_{\mu}
$$

We see that $x_{\mu}$ are the Gaussian fields with the correlation function (in the momentum space)

$$
<x_{\mu}(p) x_{\nu}(-p)>\sim \delta_{\mu \nu}|p|^{-1}
$$

Expanding the second term in $z$ we obtain

$$
S_{B} \approx \frac{1}{2} \sum|p| z(p) z(-p)+\int \nabla_{\lambda} F_{\sigma \mu}(c) \frac{d c_{\mu}}{d s} z_{\lambda}(s) z_{\sigma}(s) d s
$$

Using the fact that

$$
<z_{\lambda}(s) z_{\sigma}(s)>\sim \delta_{\lambda \sigma} \int^{\Lambda} \frac{d p}{|p|},
$$

where $\Lambda$ is an ultraviolet cut-off, we obtain as condition that the divergence cancel (in this approximation)

$$
\nabla_{\lambda} F_{\lambda \mu}=0
$$


This is the on-shell condition for the massless string mode. Qualitatively the same treatment is applicable to the massive states as well. In this case one perturbs $S_{B}$ with the operator

$$
\Delta S_{B}=\int d s \Psi(x(s))\left(\dot{x}^{2}(s) h^{-2}(s)\right)^{n} h(s),
$$

where $\Psi$ is the scalar massive mode at the level $2 n$ and $h(s)$ is the boundary metric on the world sheet needed for the general covariance of this expression. Conformal invariance of this perturbation means that the $h(s)$ dependence must cancel. The cancellation occurs between the explicit $h$-dependence in the above formula and the factors coming from the quantum fluctuations of $x(s)$. These factors appear because in the covariant theory the cut-off is always accompanied by the boundary metric

$$
\begin{gathered}
h(s)(\Delta s)_{\min }^{2}=a^{2}, \\
\Lambda^{2}=\frac{1}{(\Delta s)_{\min }^{2}}=\frac{1}{a^{2}} h(s),
\end{gathered}
$$

where $a$ is an invariant cut-off.

In the one loop approximation (which is not, strictly speaking, applicable here, but gives a correct qualitative picture) we have

$$
\begin{gathered}
\partial^{2} \Psi-M_{n}^{2} \Psi=0, \\
M_{n}^{2} \sim n .
\end{gathered}
$$

This is the on-shell condition for the massive string mode and it was obtained, let us stress it again, from the cancellation between the classical and quantum $h(s)$ dependence

Now we are ready to attack the AdS case. Let us consider the string action in this background

$$
S=\sqrt{\lambda} \int_{D} \frac{\left(\partial x_{\mu}\right)^{2}+(\partial y)^{2}}{y^{2}}+\ldots
$$

where we dropped all fermionic and RR terms. This is legitimate in the WKB limit $\lambda \gg 1$ which we will study in this section. To find the counterterms we 
must once again calculate the boundary action and $\left\langle z_{\lambda}(s) z_{\mu}(s)>\right.$. It is not as easy as in the previous case, but this well-defined mathematical problem was solved in $[4,5]$. The answer has the following form

$$
S_{c l}=\sqrt{\lambda} \int d s_{1} d s_{2} \kappa_{\mu \nu}\left(s_{1}, s_{2}\right) z_{\mu}\left(s_{1}\right) z_{\nu}\left(s_{2}\right)
$$

After introducing variables $s=\frac{s_{1}+s_{2}}{2}$ and $\sigma=s_{1}-s_{2}$ and taking the Fourier transform with respect to $\sigma$ we obtain the following asymptotic for the kernel in the mixed representation

$$
\kappa_{\mu \nu}(p, s) \underset{p \rightarrow \infty}{\approx} \frac{|p|^{3}}{\left(c^{\prime}(s)\right)^{2}}\left[3 \frac{c_{\mu}^{\prime} c_{\nu}^{\prime}}{\left(c^{\prime}\right)^{2}}-\delta_{\mu \nu}\right]
$$

¿From this it follows that

$$
<z_{\mu}(s) z_{\nu}(s)>\propto \int^{\infty} \frac{d p}{|p|^{3}}<\infty .
$$

The remarkable feature of this answer is that it implies that there is no quantum ultraviolet divergences on the world sheet. Hence, if we add to the action the background fields

$$
\Delta S \sim \int A_{\mu} d x_{\mu}+\int \Psi(x(s))\left(x^{\prime}(s)\right)^{2}(h(s))^{-1} d s+\ldots
$$

and treat it in the one loop approximation, we come to the following conclusions. First of all, as far as the $A$-term is concerned, it is finite for any $A_{\mu}(x)$ and thus describes the off-shell gluons. This situation is in the sharp contrast with the standard case in which conformal invariance implied the on-shell condition.

Now let us examine the massive mode (11). The only quantum dependence on the cut-off comes from

$$
<z_{\mu}^{\prime}(s) z_{\nu}^{\prime}(s)>\sim \int \frac{d p}{|p|}
$$

As a result we obtain the following counterterm

$$
\Delta S \sim \int \Psi(x(s))\left(x^{\prime}(s)\right)^{2}\left(\frac{\log h(s)}{h(s)}\right) d s
$$


We see that the only way to keep the theory conformally invariant in this approximation is to set $\Psi=0$. There is also a possibility that at some fixed value of $\lambda$ the $h(s)$ dependence will go away. However it is impossible to cancel it by a suitable on-shell condition. All this happens because, due to (19), the kinetic energy for the $\Psi$-term is not generated.

There is one more massless mode in the AdS string which requires a special treatment. Let us examine

$$
\Delta S=\int \Phi(x(s)) \partial_{\perp} y(s) d s
$$

where $\partial_{\perp}$ is a normal derivative at the boundary of the world sheet (which lies at infinity of the AdS space). In the more general case of $\operatorname{AdS}_{\mathrm{p}} \times \mathrm{S}_{\mathrm{q}}$ we have also a perturbation

$$
\Delta S=\int \Phi^{i}(x) n^{i}(s) \partial_{\perp} y d s
$$

Here we must remember that the string action is finite only if $[6,5]$

$$
\left(\partial_{\perp} y\right)^{2}=\left(x^{\prime}\right)^{2} \text {. }
$$

It is easy to see that when we substitute the decomposition (5) into this formula we get the logarithmic divergence (21) once again. We come to the conclusion that the above perturbation is not conformal and must not be present at the boundary. However in the case of (24) there is also a logarithmic term, coming from the fluctuations of $n^{i}(s)$. In the presence of space-time supersymmetry these two divergences must cancel since the masslessness of the scalar fields is protected by the SUSY. Otherwise, keeping the scalar fields massless requires a special fine tuning of the background. It would be interesting to clarify the corresponding mechanism.

Another interesting problem for the future is the fate of the open string tachyon in the AdS space. So far we assumed that it is excluded by the GSO projection. But in the purely bosonic string it may lead to some interesting effects via Sen mechanism [7].

We come to the following conclusion concerning the spectrum of the boundary states in the AdS-like background. It consists of a few modes which would have been massless in the flat case. The infinite tower of the 
massive states can not reach the boundary. The above finite set of states must be associated with the fields of the field theory under consideration.

The full justification of this assertion requires the analyses of the SchwingerDyson equations of the Yang-Mills theory. It is still absent, and we give some heuristic arguments instead. The loop equation, expressing the SchwingerDyson equations in terms of loops has the form

$$
\widehat{L}(s) W(C)=W * W
$$

where $W(C)$ is the Wilson loop and $\widehat{L}$ is the loop laplacian and the right hand side comes from the self-intersecting contours. In recent papers $[4$, 5] we analyzed the action of the loop Laplacian in the AdS space. It was shown that at least in the WKB approximation and in the four -dimensional space-time we have a highly non-trivial relation

$$
\widehat{L}(s) \sim T_{\perp \|}(s)
$$

where $T_{\perp \|}(s)$ is a component of the world sheet energy-momentum tensor at the boundary. When substituted inside the string functional integral the energy momentum tensor receives contributions from the degenerate metrics only. These metrics describe a pinched disk, that is two discs joined at a point. The corresponding amplitude is saturated by the allowed boundary operators inserted at this point. That gives the equation (26) provided that the boundary operators of the string are the same as the fields of the field theory. Much work is still needed to make this argument completely precise.

\section{The D-brane picture}

An alternative way to understand gauge fields-strings duality is based on the D-brane approach. It is less general than the sigma model approach described above, but in the supersymmetric cases it provides us with an attractive visual picture. The logic of this method is based on the fundamental conjecture that D-branes can be described as some particular solitons in the closed string sector. One of the strongest arguments in favor of this conjecture is that both D-branes and solitons have the same symmetries and are sources of the same RR fields [8]. The gauge fields -strings dualities then follows from the D-branes - solitons duality in the limit $\alpha^{\prime} \rightarrow 0$. On the Dbrane side only the massless gauge field modes of the open string survive in 
this limit. On the string theory side we have a near horizon limit [1] of the soliton metric [9] , given by (1). These two theories must be equivalent, if the basic D-brane conjecture is correct.

The connection with the sigma model approach of the previous section follows from the following argument. First of all the closed string background is the same in both cases. As for the open strings, we placed their ends at the boundary of the AdS space where $a^{2}(\varphi) \rightarrow \infty$. That means that the effective slope of this strings behaves as

$$
\alpha_{\text {open }}^{\prime} \sim a^{-2}(\varphi) \rightarrow 0
$$

and thus only the massless modes are present. We said that the D-brane approach is less general, because in the non-supersymmetric cases there could exist solitons with the required boundary behavior, which are not describable by any combination of D-branes in the flat space.

This fact is related to another often overlooked subtlety. The 3-brane soliton has a metric [9]

$$
\begin{aligned}
d s^{2} & =H^{-\frac{1}{2}}(r)(d x)^{2}+H^{\frac{1}{2}}(r)(d y)^{2} \\
r^{2} & =y^{2} ; \quad H(r)=1+\frac{L^{4}}{r^{4}}
\end{aligned}
$$

It is often assumed that this metric is an extremum of the action

$$
S=S_{b u l k}+S_{B I}
$$

where the first term contains the modes of the closed string, while the second is the Born -Infeld action localized on the brane. In the equations of motion the second term will give a delta function of the transverse coordinates.

Would it be the case, where the 3-brane is located? From (30) it is clear that the singularity of the metric is located in the complex domain $r^{4}=-L^{4}$. Let us try to understand the significance of this fact from the string-theoretic point of view. Consider a string diagram describing the Dbrane world volume in the arbitrary order in $\lambda=g_{s} N$. It is represented by a disc with an arbitrary number of holes. At each boundary one imposes the Dirichlet conditions for the transverse coordinates. An important feature of this diagram is that it is finite. This follows from the fact that the only source of divergences in string theory are tadpoles and for 3-branes their 
contribution is proportional to the integral $\int \frac{d^{6} k}{k^{2}}$ where $k$ is the transverse momentum. This expression is infrared finite (which is of course very well known). Thus D-branes in the flat space are described by the well defined string amplitudes. But that contradicts the common wisdom, that one must determine the background from the action (31), because the flat space is not a solution, once the Born-Infeld term is added. More over if we try to deform the flat space, the above disc with holes will loose its conformal invariance.

Let us analyze this apparent paradox. It is related to the fact on a sphere conformal invariance is equivalent to the absence of tadpoles since for any $(1,1)$ vertex operator we have $\langle V\rangle_{\text {sphere }}=0$. However on a disc this is not true, the conformal symmetry doesn't forbid the expectation values of vertex operators. On a disc conformal symmetry and the absence of tadpoles are two different conditions. Which one should we use?

If we denote the bulk couplings by $\lambda$ and the boundary couplings by $\mu$ we can construct three different objects, the bulk central charge $c(\lambda)$, the "boundary entropy" [10] $b(\lambda, \mu)$ and the effective action generating the Smatrix, $S(\lambda, \mu)=c(\lambda)+b(\lambda, \mu)$. To ensure conformal invariance we must have

$$
\begin{aligned}
& \frac{\partial c}{\partial \lambda}=0 \\
& \frac{\partial b}{\partial \mu}=0
\end{aligned}
$$

This does not coincide in general with the "no tadpole condition"

$$
\frac{\partial S}{\partial \lambda}=\frac{\partial S}{\partial \mu}=0
$$

In the case of 3-branes the paradox is resolved in an interesting way. The metric (30) has a horizon at $r=0$. When we go to the Euclidean signature the horizon, as usual, shrinks to a non-singular point. As a result we have a metric which solves the equation ( 32 ) and has no trace of the D-brane singularity in it! The paradox is pushed under the horizon.

The conclusion of this discussion is as following. We have two dual and different descriptions of the D-brane amplitudes. In the first description we calculate the amplitudes of a disc with holes in the flat space. In this description it is simply inconsistent to introduce the background fields generated by D-branes. 
In the second description we forget about the D-branes and study a nonsingular closed string soliton. The D-brane conjecture implies that we must get the same answers in these two cases. The situation is analogous to the one we have in the sine-gordon theory, which admits two dual descriptions, either in terms of solitons or in terms of elementary fermions, but not both.

Let us touch briefly another consequence of these considerations. When minimizing the action ( 31 ) one can find a solution which is singular on the 3-brane and is AdS-space outside of it [11]. These solutions are known to "localize" gravitons on the brane and are the basis of the popular "braneworld" scenarios. It is clear that for the string-theoretic branes this is not a physical solution because the world volume does not contain gravity (being described by the open strings). As we argued above, there must be a horizon, not a singularity. Technically this happens because in string theory the BornInfeld action is corrected with the Einstein term $\sim \int R \sqrt{g} d^{p+1} x$, coming from the finite thickness of the brane. It can be shown that the coefficient in front of this term (which is fully determined by string theory) is tuned so that the localization is destroyed. There are no worlds on D-branes. Of course if one compactifies the ambient transverse space, the $4 \mathrm{~d}$ graviton reappears by the Kaluza-Klein mechanism.

\section{Conformal gauge theories in higher dimen- sions}

Although our main goal is to find a string theoretic description of the asymptotically free theories, conformal cases are not without interest. They are easier and can be used as a testing ground for the new methods. In this section we briefly discuss conformal bosonic gauge theories in various dimensions [2 ]. The background in these cases is just the AdS space. We have to perform the non-chiral GSO projection in order to eliminate the boundary tachyon (it would add an instability to the field theory under consideration; we do not consider here an interesting possibility that this instability resolves in some new phase).

The GSO projection in the non-critical string is slightly unusual. Let us consider first $d=5$ (corresponding to the $d=4$ gauge theory). In this case we have 4 standard NSR fermions $\psi_{\mu}$ on the world sheet and also a partner of the Liouville field $\psi_{5}$. For the former we can use the standard spin fields 
defined by the OPE

$$
\psi_{\mu} \times \Sigma_{A} \sim\left(\gamma_{\mu}\right)_{A B} \Sigma_{B}
$$

where we use the usual $4 \times 4$ Dirac matrices. The spinor $\Sigma_{A}$ can be split into spinors $\Sigma_{A}^{+}$with positive and negative chiralities. It is easy to check that the OPE for them have the structure

$$
\begin{aligned}
& \Sigma^{+} \times \Sigma^{+} \sim(\psi)^{[\text {even }]} \\
& \Sigma^{+} \times \Sigma^{-} \sim(\psi)^{[\text {odd }]}
\end{aligned}
$$

The symbols on the RHS mean the products of even/odd number of the NSR fermions. Notice that this structure is the opposite to the one in 10 dimension. In order to obtain the spin operators in $5 \mathrm{~d}$ we have to introduce the Ising order and disorder operators , $\sigma$ and $\mu$, related to $\psi_{5}$. These operators are non-holomorphic and correspond to RR-states. Their OPE have the structure

$$
\begin{aligned}
\sigma \times \sigma & \sim\left(\psi_{5}\right)^{[\text {even }]} \\
\sigma \times \mu & \sim\left(\psi_{5}\right)^{[\text {odd }]}
\end{aligned}
$$

Using these relations we obtain the following GSO projected RR spin operator

$$
\Sigma=\left(\begin{array}{ll}
\sigma \Sigma^{+} \overline{\Sigma^{+}} & \mu \Sigma^{+} \overline{\Sigma^{-}} \\
\mu \Sigma^{-} \overline{\Sigma^{+}} & \sigma \Sigma^{-} \overline{\Sigma^{-}}
\end{array}\right)
$$

It has the property

$$
\Sigma \times \Sigma \sim(\psi)^{[e v e n]}
$$

needed for the non-chiral GSO projection, which consists of dropping all operators with the odd number of fermions. Notice also that the non-chiral picture changing operator has even number of fermions. The RR matrix $\Sigma$ has 16 elements. We can now write down the full string action in the $\mathrm{AdS}_{5}$ space. It has the form

$$
S=S_{B}+S_{F}+S_{R R}+S_{g h o s t}
$$


where $S_{B}$ is given by $(16), S_{F}$ by

$$
S_{F}=\int d^{2} \xi\left[\bar{\psi}_{M} \nabla \psi_{M}+\frac{1}{\sqrt{\lambda}}\left(\overline{\psi_{M}} \gamma_{\mu} \psi_{N}\right)^{2}\right]
$$

where $M=1, \ldots 5$, and one have to use the standard spin-connection projected from AdS on the world sheet in the Dirac operator. So far we are describing the usual action of the sigma model with $N=1$ supersymmetry on the world sheet. The unusual part is the RR term given by

$$
S_{R R}=f \int d^{2} \xi \operatorname{Tr}\left(\gamma_{5} \Sigma\right) e^{-\frac{\phi}{2}}
$$

Here $e^{-\frac{\phi}{2}}$ is a spin operator for the bosonic ghost [12 ] and $f$ is a coupling constant (which in one loop approximation is equal to 1 ).

I believe that this model can be exactly solved, although it has not been done yet. A promising approach to this solution may be based on the nonabelian bosonization [13] in which the fermions $\psi_{M}$ are replaced by the orthogonal matrix $\Omega_{M N}$ with the WZNW Lagrangian. In this case the RR term is simply a trace of this matrix in the spinor representation. This formalism lies in the middle between the NSR and the Green-Schwartz approaches and hopefully will be useful. Meanwhile we will have to be content with the one loop estimates which are justified in some special cases listed below and help to get a qualitative picture in general. In this approach one begins with the effective action

$$
S=\int d^{d} x \sqrt{G} e^{\Phi}\left[\frac{d-10}{2}-R-(\nabla \Phi)^{2}\right]+\int d^{d} x F_{d}^{2} \sqrt{G}
$$

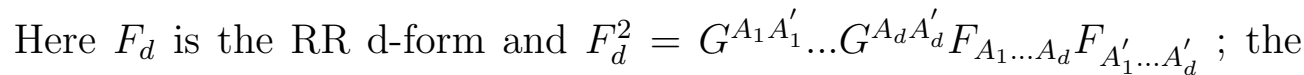
form $F_{d}$ will be assumed to be proportional to the volume form . The dilaton field $\Phi$ is normalized so that $e^{\Phi}=g_{s}^{-2}$, where $g_{s}$ is the string coupling constant. Conformal cases involve either constant curvature solutions of the equations of motion or the products of the manifolds with constant curvatures. The dilaton in these cases is also a constant. Such an ansatz is very easy to analyze. Let us begin with the single $\mathrm{AdS}_{d}$ space. Consider the variation of the metric which preserves the constancy of the curvature

$$
\begin{aligned}
\delta G_{A B} & =\varepsilon G_{A B} \\
\delta R & =\delta\left(G^{A B} R_{A B}\right)=-\varepsilon R \\
\delta \Phi & =\text { const }
\end{aligned}
$$


That immediately gives the relations

$$
\begin{array}{r}
\frac{d-10}{2}-R=0 \\
e^{\Phi}\left(1-\frac{d}{2}\right) R-\frac{d}{2} F_{d}^{2}=0
\end{array}
$$

If the assume that the flux of the RR field is equal to $N$, we get $F_{d}^{2}=N^{2}$. If we introduce the coupling constant $\lambda=g_{s} N=g_{Y M}^{2} N$, we obtain the background AdS solution [2] with

$$
|R| \sim \lambda^{2} \sim 10-d
$$

We can trust this solution if $d=10-\epsilon$, in which case the curvatures and the RR fields are small and the above one loop approximation is justified. According to the discussion in the preceding sections, this solution must describe the Yang-Mills theory, perhaps with one adjoint scalar, in the space with dimension $9-\epsilon$. We conclude that this bosonic higher dimensional gauge theory has a conformally invariant fixed point! It may be worth mentioning that this is not atypical for non-renormalizable theories to have such fixed points. For example a nonlinear sigma model in dimension higher than two, where it is non-renormalizable, does have a conformal critical point at which the phase transition to ferromagnetic phase takes place. However it is hard to say up to what values of $\epsilon$ we can extrapolate this result.

These considerations allow for several generalizations. First of all we can consider products of spaces with constant curvatures by the same method. Take for example the space $\mathrm{AdS}_{p} \times S_{q}$ with curvatures $R_{1}$ and $R_{2}$ and $d=p+q$. To get the equations of motion in this case it is sufficient to consider the variations

$$
\begin{aligned}
\delta G_{a b} & =\varepsilon_{1} G_{a b} \\
\delta G_{i j} & =\varepsilon_{2} G_{i j}
\end{aligned}
$$

where the first part refers to AdS and the second to the sphere. A simple 
calculation gives the equations

$$
\begin{aligned}
\frac{d-10}{2}-R_{1}-R_{2}= & 0 \\
& \left(1-\frac{2}{p}\right) R_{1}+\frac{10-d}{2}=-e^{-\Phi} F_{d}^{2} \\
\left(1-\frac{2}{q}\right) R_{2}+\frac{10-d}{2}= & e^{-\Phi} F_{d}^{2} \\
F_{d}^{2}= & \lambda^{2} R_{2}^{q}
\end{aligned}
$$

Here we assume that the RR flux is permeating the AdS component of space only, being given by the volume form. The last equation follows from the normalization condition of this flux and the extra factor proportional to the volume of $S_{q}$ in the action. Solving this equations we get

$$
\begin{aligned}
& R_{1}=-\left(\frac{10-p-q}{2}\right) \frac{p(q+2)}{p-q} \\
& R_{2}=\left(\frac{10-p-q}{2}\right) \frac{q(p+2)}{p-q}
\end{aligned}
$$

This solution describes bosonic gauge theories with $q+1$ adjoint bosons; again it can be trusted if the curvatures are small. Another generalization is related to the fact that strictly speaking we must include the closed string tachyon in our considerations. It was shown in [14] that there exists an interesting mechanism for the tachyon condensation, following from its couplings to the RR fields. It is easy to include the constant tachyon field in our action and to show that it doesn't change our results in the small curvature limit. According to [14], in the critical case $d=10$ the tachyon leads to the running coupling constants. In the non-critical case there is also a conformal option, described above, in which the tachyon condenses to a constant value.

Finally let us describe the reasons to believe that the conformal solutions can be extrapolated to non-small curvatures and thus the sigma model (42) has a conformal fixed point. The first two terms in (45) are the expansion of the sigma model central charge. When the couplings are not small, we have to replace

$$
\frac{d-10}{2}-R \Rightarrow \frac{c(R)-10}{2}
$$


where $c(R)$ is the the central charge of the $2 \mathrm{~d}$ sigma model with the target space having a curvature $R$. It decreases, according to Zamolodchikov, along the renormalization group trajectory. We can call this the second law of the renormalization group. Let us conjecture that there is also a third law

$$
\begin{aligned}
& c(R) \rightarrow 0(R \rightarrow+\infty) \\
& c(R) \rightarrow \infty(R \rightarrow-\infty)
\end{aligned}
$$

The first property follows from the fact that usually the sigma models with positive curvature develop a mass gap and thus there are no degrees of freedom contributing to the central charge. The second equation is harder to justify; we know only that $c(R)$ is increasing in the direction of negative curvature.

With this properties and with some general form of the RR terms it is possible to see that the conformal solution to the equations of motion, obtained by the variations ( 46 ), continue to exist when $\epsilon$ is not small. Of course this is not a good way to explore these solutions. Instead one must construct the conformal algebra for the sigma model action(42). This has not been done yet.

\section{Infrared screening of the cosmological con- stant and other speculations}

In this section we will discuss some speculative approaches to the problems of vacuum energy and space-time singularities. I shall try to revive some old ideas [15], adding some additional thoughts. The motivation to do that comes from the remarkable recent observational findings indicating that the cosmological constant is non- zero, and its scale is defined by the size of the universe (meaning the Hubble constant). These results seem very natural from the point of view advocated in [15], according to which there is an almost complete screening of the cosmological constant due to the infrared fluctuations of the gravitational field. This phenomenon is analogous to the complete screening of electric charge in quantum electrodynamics found by Landau, Abrikosov and Khalatnikov and nicknamed "Moscow zero". Here we will try to argue in favor for another "zero" of this kind -that of the cosmological constant. 
Let us consider at first the Einstein action

$$
S=-\int\left(R-2 \Lambda_{0}\right) \sqrt{g} d^{4} x
$$

Here $\Lambda_{0}$ is a bare cosmological constant which is assumed to be defined by the Planck scale. It is clear from the form of the action that if we consider the infrared fluctuations of the metric (with the wave length much larger then the Planck scale) their interaction will be dominated by the second term in this formula since it doesn't contain derivatives. To get some qualitative understanding of the phenomenon, let us consider conformally flat fluctuations of the metric

$$
g_{\mu \nu}=\varphi^{2} \delta_{\mu \nu}
$$

The action takes the form

$$
S=-\int\left[\frac{1}{2}(\partial \varphi)^{2}-\Lambda_{0} \varphi^{4}\right] d^{4} x
$$

It has the well known feature of non-positivity. The way to treat it was suggested in [16] and we will accept it, although it doesn't have good physical justification. To use $S$ in the functional integral we will simply analytically continue $\varphi \Rightarrow i \varphi$; after that the action takes the form

$$
S=\int\left[\frac{1}{2}(\partial \varphi)^{2}+\Lambda_{0} \varphi^{4}\right] d^{4} x
$$

The infrared fluctuations of $\varphi$ are relevant and lead to the screening of $\Lambda_{0}$

$$
\Lambda \sim \frac{1}{\log \left(M_{p l} L\right)}
$$

where $L$ is an infrared cut-off. More generally we could represent the metric in the form

$$
g_{\mu \nu}=\varphi^{2} h_{\mu \nu} ; \operatorname{det}\left(h_{\mu \nu}\right)=1
$$

It is not known how to treat the unimodular part of the metric. We can only hope that it will not undo the infrared screening although can change it. Also the screening (67) with $L \sim H^{-1}$ (where $H$ is the Hubble constant ) is 
not strong enough to explain the fact that $\Lambda \sim H^{2}$. It is not impossible that the two problems cure each other. When we have several relevant degrees of freedom, the renormalization group equations governing the $L$ dependence of various coupling constants including $\Lambda$ may have a powerlike asymptotic (in contrast with $(67)$ ). Such examples exist, starting from the cases with two independent coupling constants. Thus the infrared limit of the Einstein action (perhaps with the dilaton added ) may be described by a conformal field theory, giving the cosmological constant defined by the Hubble scale. The renormalization group should take us from Planck to Hubble.

Even if this fantasy is realized, we have to resolve another puzzle. It is certainly unacceptable to have a large cosmological constant in the early Universe, since it will damage the theory of nucleosynthesis. At the first glance it seems to create a serious problem for the screening theory,because when the universe is relatively small, the screening is small also and the cosmological constant is large. The way out of this problem is to conjecture that in the radiation dominated universe the infrared cut-off is provided by the curvature of space-time, while in the matter-dominated era it begins to depend on other quantities characterizing the size of the universe. If this is the case, in the early universe we get the screening law $\Lambda \sim R$ instead of $\Lambda \sim H^{2}$. Substituting it in the Einstein action we find that at this stage the infrared mechanism simply renormalizes the Newton constant and thus is unobservable. In the matter dominated era the effective $\Lambda$ begin to depend on other things (like the Friedman warp factor $a$ ) and that can easily give the observed acceleration of the universe. Thus the change in the infrared screening must be related to a trace of the energy momentum tensor. We can say that in the correct theory the cosmological constant vanishes without the trace. To be more precise, it is disguised as a Newton constant, until the trace of the energy-momentum tensor reveals its true identity. The above picture have some remote resemblance to the scenario suggested recently in [17].

In spite of the obvious gaps in these arguments, they give a very natural way of relating the cosmological constant to the size of the universe and thus are worth developing. Perhaps the AdS/CFT correspondence will be of some use for this purpose. The main technical problem in testing these ideas is the unusual $\varphi$ - dependent kinetic energy of the $h$-field.

We can also notice that the above scenario can explain the dimensionality of space-time. Indeed, if this dimensionality is larger then four, the infrared effects are small, the cosmological constant- large, and we end up in the 
universe of the Planck size, which is not much fun.

This work was supported in part by the NSF grant PHY9802484. 


\section{REFERENCES}

[1] O. Aharony, S.Gubser, J. Maldacena, H. Ooguri, Phys. Rep. 323 (2000) 183

[2] A. Polyakov J. Mod. Phys. A14 (1999) 645, hep-th/9809057

[3] C. Callan et al. Nucl. Phys. B308 (1988) 221

[4] A. Polyakov, V. Rychkov hep-th/0002106

[5] A. Polyakov, V. Rychkov hep-th/0005173

[6] N. Drukker, D. Gross, H. Ooguri Phys. Rev.D60 (199) 125, hepth/9904191

[7] A. Sen JHEP 9912027, hep-th/9911116

[8] J. Polchinski Phys. Rev. Lett. 75 (1995) 4724, hep-th/9510017

[9] G. Horowitz, A. Strominger Nucl. Phys. B360 (1991) 197

[10] I. Affleck, A. Ludwig Phys.Rev.Lett. 67 (1991) 161

[11] L. Randall, R. Sundrum Phys. Rev. Lett. 83 (1999) 4690

[12] D. Friedan, E. Martinec, S. Shenker Nucl. Phys.B271 (1986) 93

[13] E. Witten Comm. Math. Phys.92 (1984) 455

[14] Ig. Klebanov A. Tseytlin Nucl. Phys.B547 (1999) hep-th/9812089

[15] A. Polyakov Sov. Phys. Uspekhi 25 (1982) 187

[16] G. Gibbons, S. Hawking Phys.Rev.D15 (1977) 2725

[17] C. Armendariz-Picon, V. Mukhanov, P. Steinhardt, astro-ph/0004139 